Provided for non-commercial research and education use. Not for reproduction, distribution or commercial use.

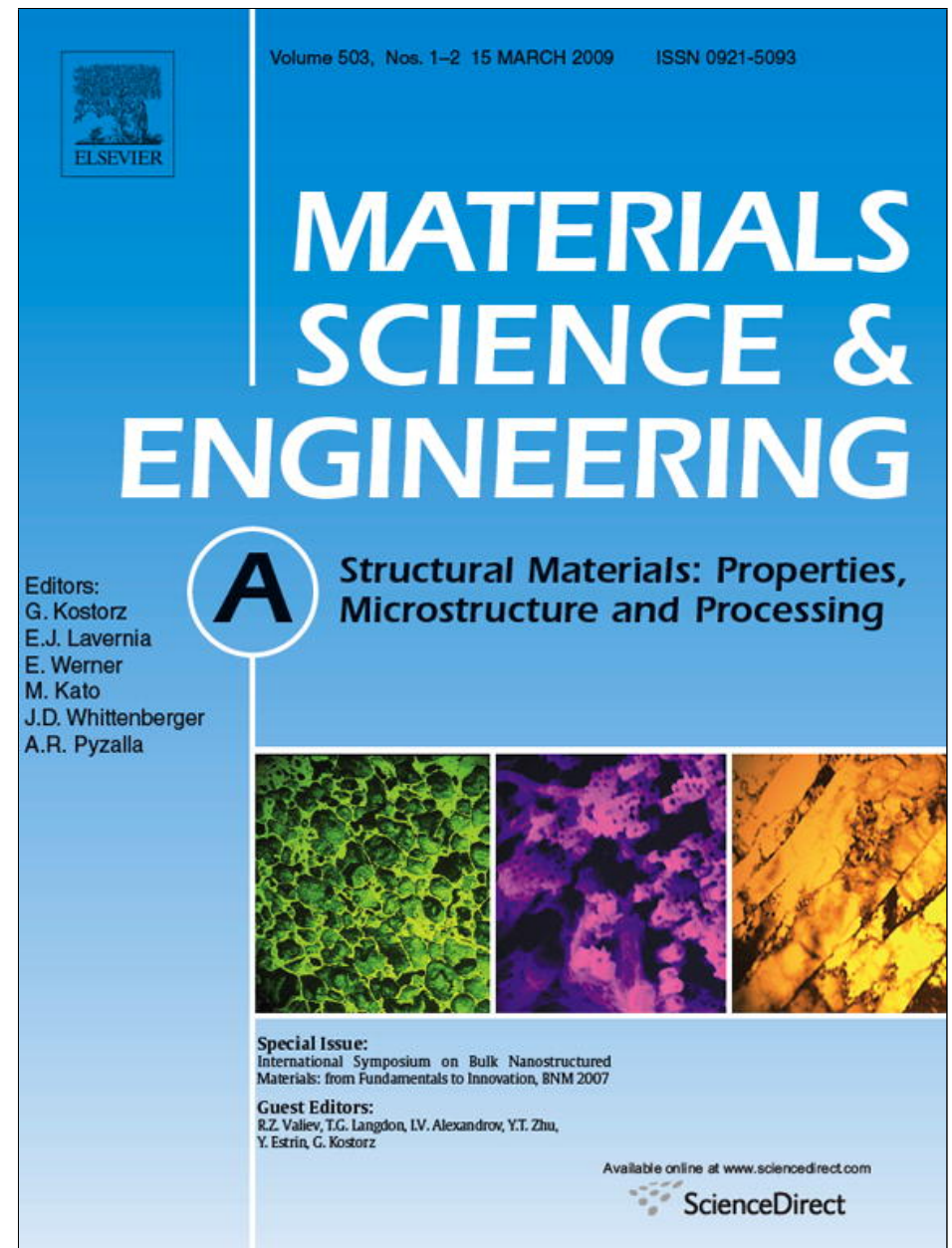

This article appeared in a journal published by Elsevier. The attached copy is furnished to the author for internal non-commercial research and education use, including for instruction at the authors institution and sharing with colleagues.

Other uses, including reproduction and distribution, or selling or licensing copies, or posting to personal, institutional or third party websites are prohibited.

In most cases authors are permitted to post their version of the article (e.g. in Word or Tex form) to their personal website or institutional repository. Authors requiring further information regarding Elsevier's archiving and manuscript policies are encouraged to visit:

http://www.elsevier.com/copyright 


\title{
Useful properties of twist extrusion
}

\author{
Y. Beygelzimer*, V. Varyukhin, S. Synkov, D. Orlov \\ Donetsk Institute of Physics \& Engineering of the NAS of Ukraine, 72 R. Luxemburg St., Donetsk 83114, Ukraine
}

\section{A R T I C L E I N F O}

\section{Article history:}

Received 17 October 2007

Received in revised form 17 December 2007

Accepted 20 December 2007

\section{Keywords:}

Twist extrusion

Severe plastic deformation

Ultrafine-grained materials

\begin{abstract}
A B S T R A C T
We present an experimental study of the kinematics of twist extrusion (TE) and show that TE has the following properties: (i) as in equal-channel angular pressing (ECAP), the mode of deformation in twist extrusion is simple shear. Unlike in ECAP, there are two shear planes; one of them is perpendicular and the other is parallel to the specimen axis. (ii) The following processes are present during twist extrusion: vortex-like flow with large strain gradient, stretching and mixing of metal particles. We argue that, due to these properties, TE opens possibilities for investigating and forming new microstructures. It has already been successfully used to obtain ultrafine-grained microstructures with good properties in $\mathrm{Al}, \mathrm{Cu}$ and $\mathrm{Ti}$ alloys.
\end{abstract}

(C) 2008 Elsevier B.V. All rights reserved.

\section{Introduction}

Severe plastic deformation (SPD) is a family of metal forming techniques that use extensive hydrostatic pressure to impose a very high strain on bulk solids, producing exceptional grain refinement without introducing any significant change in the overall dimensions of the sample [1]. Several different SPD processing techniques are now available including high-pressure torsion (HPT) [2], equalchannel angular pressing (ECAP) [3], multi-directional forging (MDF) [4], accumulative roll-bonding (ARB) [5], repetitive corrugation and strengthening (RCS) [6] and twist extrusion (TE) [7,8].

Each process has unique properties determining its use in research and practice. This paper presents several properties of $\mathrm{TE}$, which open possibilities for investigating and forming new microstructures.

\section{Basics of twist extrusion}

TE is based on pressing out a prism specimen through a die with a profile consisting of two prismatic regions separated by a twist part (Fig. 1). As the specimen is processed, it undergoes severe deformation while maintaining its original cross-section. This property allows the specimen to be extruded repeatedly in order to accumulate the value of deformation, which changes in the microstructure and properties of the specimen.

TE is performed under high hydrostatic pressure in the center of deformation. The pressure is created by applying backpressure to the specimen when it exits the die.

\footnotetext{
* Corresponding author. Tel.: +38 6233776 08; fax: +38 623377608 .

E-mail addresses: yanbeygel@gmail.com, tean@an.dn.ua (Y. Beygelzimer).
}

\section{Experimental investigation of TE kinematics}

To experimentally study the kinematics of metal flow during TE, we use a specimen with nine fibers embedded along its main axis. ${ }^{1}$ The specimen was pressed through a built-up twist die until stationary flow was reached. It was then removed from the die and cut perpendicularly to its main axis with an interval of $0.5 \mathrm{~mm}$, starting from its end. The nine markers in the obtained cross-sections (shown schematically in Fig. 1) are used to reconstruct the corresponding experimental lines of flow, which are then used to fit a theoretical model of the velocity field $[8,9]$. The model incorporates two physical constraints: (1) metal flow is limited by the surface of the die and (2) metal volume remains constant. The parameters of this model are determined from fitting the observed experimental streamlines. The obtained velocity field is then used to find the strain state of the metal using the continuum mechanics relations.

We performed the experiments on $\mathrm{Al}, \mathrm{Cu}, \mathrm{Ti}$, and their alloys. All of them have common, characteristic deformation properties described next.

\section{Two shear planes and four deformation zones in TE}

There are four sufficiently well separated deformation zones observed when processing different materials with twist extrusion.

Deformation zones 1 and 2 are located at the two ends of the twist part of the die (see Fig. 1). The mode of deformation in these zones is simple shear in the transversal plane (TP), as in HPT. The shears in the two zones have opposite directions, since the prism

\footnotetext{
1 Dr. A. Reshetov, Ing. O. Prokof'eva, Ing. R. Kulagin contributed to the experimental
} part. 


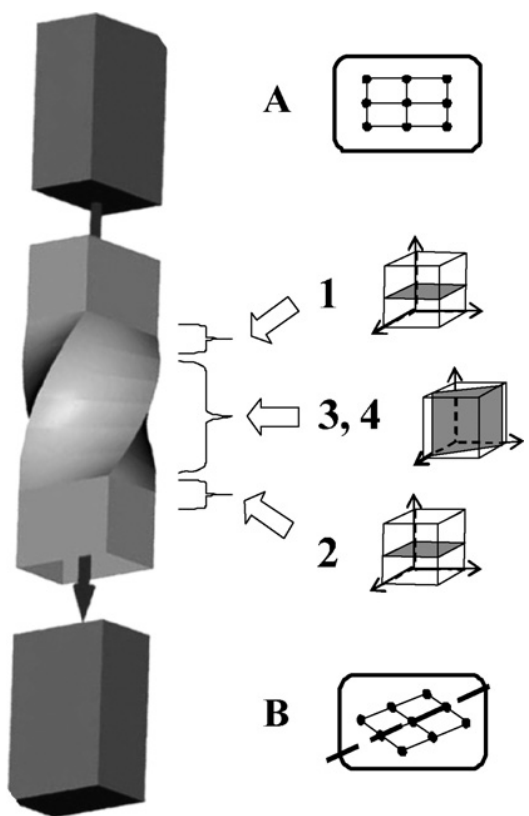

Fig. 1. Twist extrusion scheme. The numbers 1-4 show the position of the four deformation zones with the orientation of their respective shift planes. The markers in cross-sections A and B are placed on a grid to better illustrate the shift occurring in zone 3.

specimen is twisted in zone 1 and straightened in zone 2 being restored to its original shape. Each zone gives a von Mises equivalent strain from $e \sim 0.0$ (in the center) to $e \sim 0.5-0.7$ (on the periphery). The zones appear as steps in Fig. 2, which shows how the equivalent strain depends on the coordinate along the extrusion axis.

Deformation zone 3 is located in the twist part of the die between zones 1 and 2 and comprises most of the specimen volume except for a peripheral layer 1-2 mm thick. The deformation mode in zone 3 is simple shear in the rotating longitudinal plane (LP), which is indicated by the position of the markers in the crosssections (cross-section $B$ in Fig. 1). The value of equivalent strain accumulated in this zone is in the range 0.2-0.4. It is this zone that provides the deformation along the extrusion axis, where the equivalent strain in zones 1 and 2 is zero (Fig. 2).

Deformation zone 4 is located in the twist part of the die in the peripheral layer (1-2 mm thick) of the specimen between zones 1 and 2 (Fig. 1). The mode of deformation in this zone is severe simple shear with an equivalent strain of $e \sim 2$ along the cross-section contour (Fig. 2).

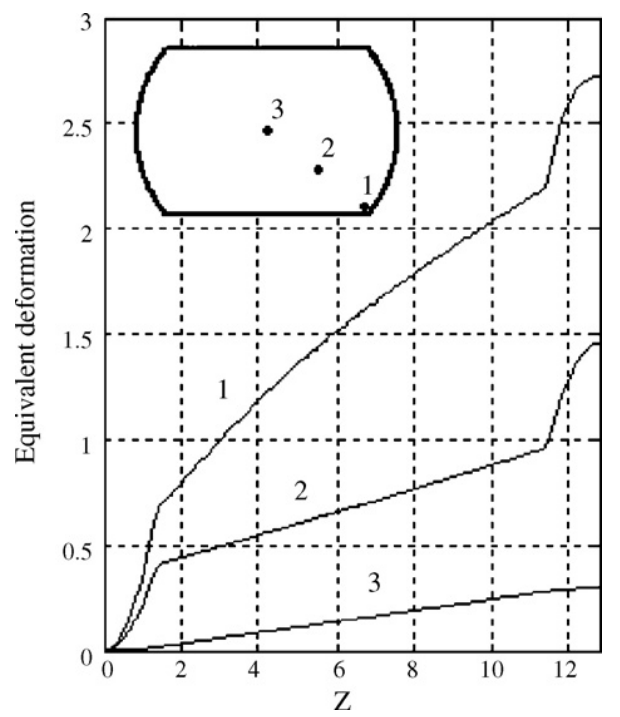

Fig. 2. Equivalent strain versus coordinate along the extrusion axis $(Z)$ for representative points of the specimen cross-section, $\beta=55^{\circ}, \alpha=70^{\circ}$.

\section{Two main routes of TE}

There are two types of twist dies: clockwise (CD) and counterclockwise (CCD). When transitioning from $C D$ to $C C D$, the shears in each of the four deformation zones reverse its sign. This gives us two main routes of TE:

Route I: $\mathrm{CD}+\mathrm{CD}$ ( or $\mathrm{CCD}+\mathrm{CCD})$,

Route II: $\mathrm{CD}+\mathrm{CCD}$ ( or CCD + CD).

Fig. 3 schematically shows the sign-sensitive change in the shift deformation along the transversal $\left(\gamma_{\mathrm{T}}\right)$ and the longitudinal $\left(\gamma_{\mathrm{L}}\right)$ planes during TE along routes I and II. It is easy to see that in the transversal plane, route 1 gives a cyclic deformation with some amplitude $A$, while route II gives a cyclic deformation with amplitude $2 \mathrm{~A}$. In the longitudinal plane, route I gives a monotonic deformation while route II gives a cyclic deformation. Different loading paths can lead to different structures and properties.

\section{Structure and properties during multi-pass TE}

Refs. [10-16] present experimental results with TE for different materials ( $\mathrm{Al}, \mathrm{Cu}, \mathrm{Ti}$, and their alloys; powders of different composition). Using optical microscopy, it is shown that cross-sections

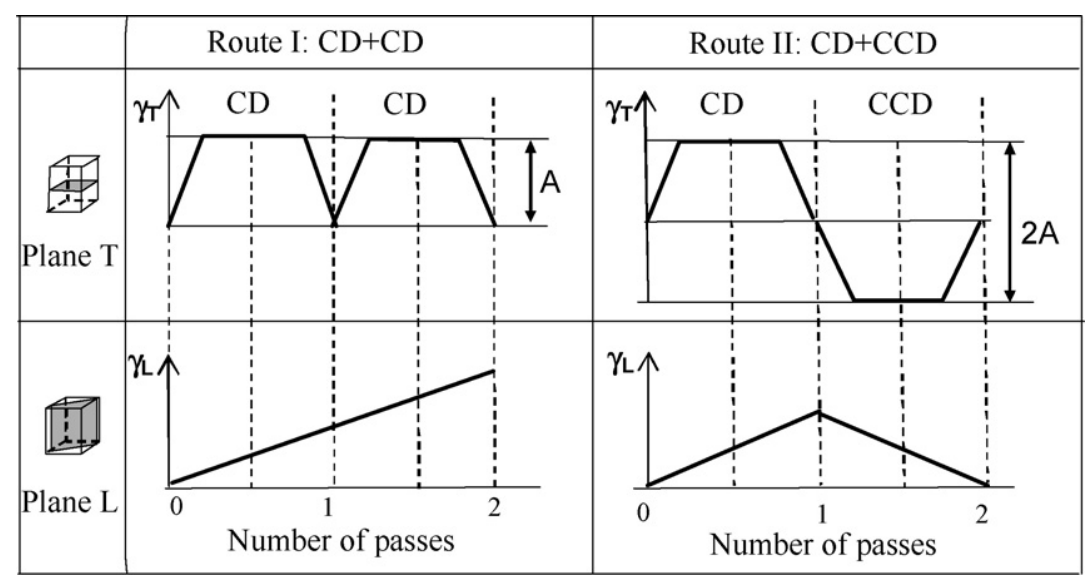

Fig. 3. Sign-sensitive change in the shift deformation along the transversal plane $\left(\gamma_{\mathrm{T}}\right)$ and the longitudinal plane $\left(\gamma_{\mathrm{L}}\right)$ during twist extrusion along routes I and II. 
(a)

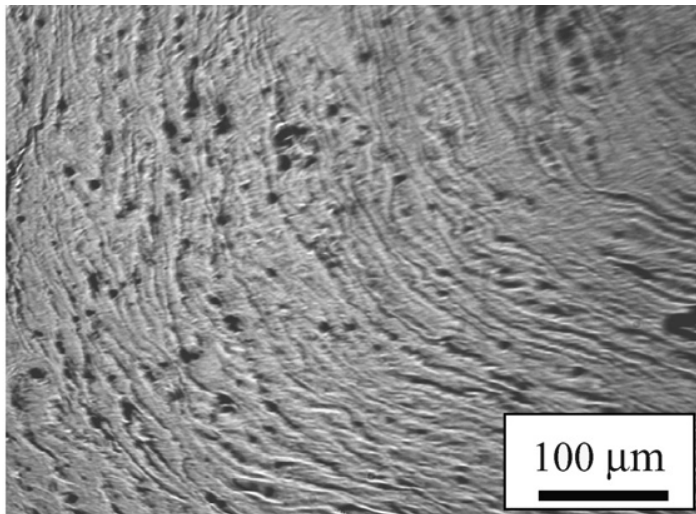

(b)

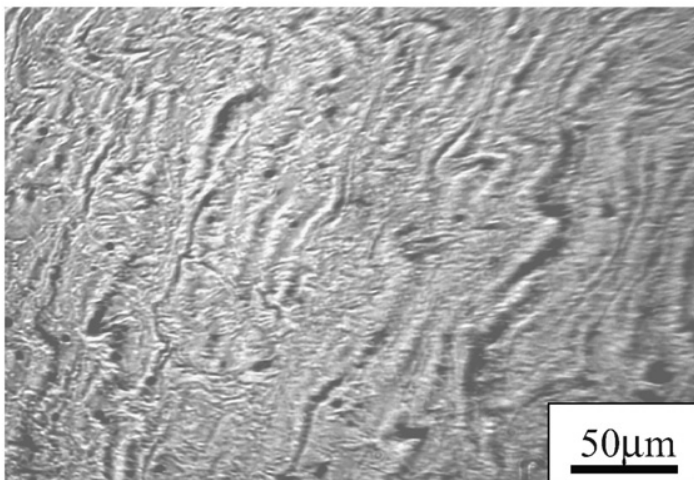

(c)

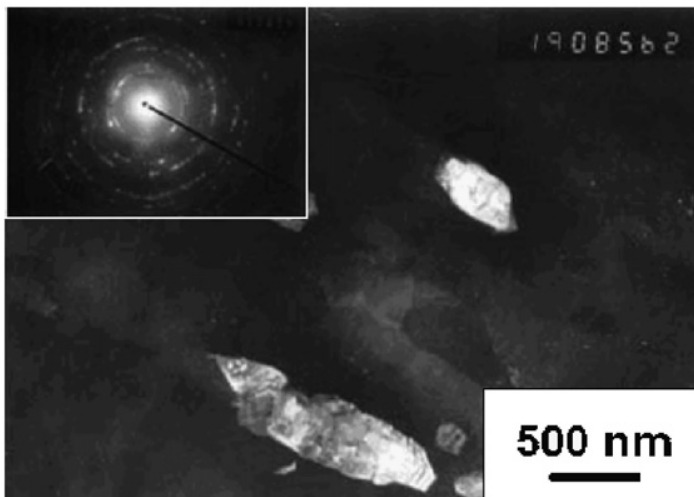

Fig. 4. Structures formed in the Al-Mg-Sc-Zr alloy processed with TE. (a, b) Optical micrographs, (c) transmission electron microscopy, dark field images and selectedarea diffraction pattern; (b) shows folds due to a severe shear in zone 4 . The electron and optical microscopies were performed in Dr. Milman's laboratory (Frantcevych Institute for Problems of Materials Science, Kiev, Ukraine).

typically exhibit a characteristic macrostructure with structural elements elongated along the direction of a vortex centered at the extrusion axis. In the longitudinal cross-section this macrostructure resembles a turbulent flow $[9,13]$. The underlying microstructure after multiple TE passes is characterized by submicrometer sizes and high angular boundaries [11,13-16]. Fig. 4 should clarify these observations. This structure provides good strength and ductility properties in the specimens processed with TE. In some cases the properties are strongly anisotropic [9,13], but the anisotropy can be obviated by changing the form of the die cross-section, increasing the operating temperature, subsequent recrystallizing thermal processing or such post-processing deformations as drawing or direct extrusion. With that, the microstructure still remains submicrocrystalline $[9,13]$.

Despite the inhomogeneity of deformation, the microstructure and properties of materials tend to even out with subsequent TE (a)

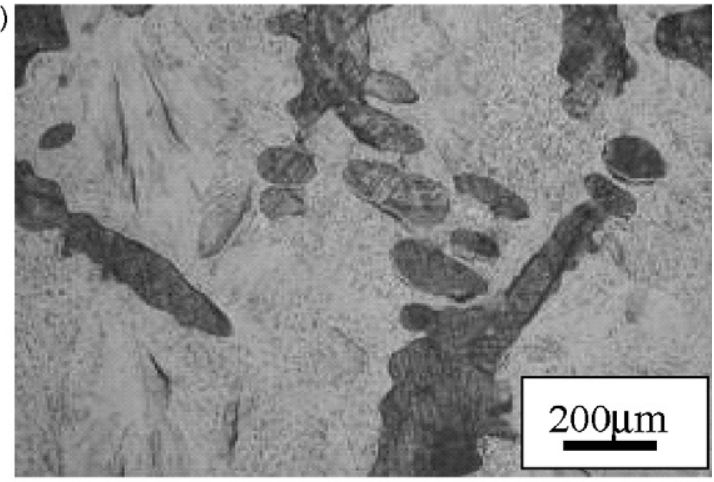

(b)

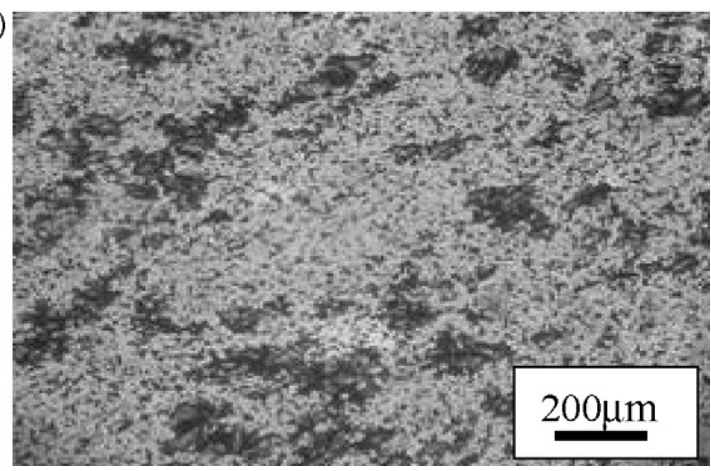

Fig. 5. Homogenization of copper phosphide $\mathrm{Cu}_{3} \mathrm{P}$ during warm twist extrusion of $\mathrm{Cu}-\mathrm{P}$ alloy: (a) initial state and (b) after 2 pass TE, route II.

passes. This is due to two factors: (1) mixing (see below) and (2) stabilization of microstructure and saturation of properties once strain exceeds some saturation level $e_{\mathrm{s}}[10,16]$. Such stabilization and saturation are not unique to TE. They are typically present in any deformation method based on simple shear; for example, saturation happens in torsion [17]. A possible mechanism explaining this effect in SPD is analyzed in Ref. [18]. With the number of passes, the zone where strain exceeds the saturation threshold, gradually fills up the entire cross-section. This tends to level out the microstructural variations and properties across the cross-section [10].

\section{New possibilities coming from TE}

The main routes of TE can be combined with any SPD or metal forming processes (e.g., ECAP, rolling, and extrusion) to broaden the space of possible loading paths. The field of equivalent strain under TE has a large gradient. This is of interest for investigating the effects of strain gradient on the evolution of the material's microstructure, as well as obtaining microstructural gradients.

Strain distribution and deformation zone boundaries strongly depend on the geometry of the die cross-section, inclination angle of the twist line $\beta$ and rotation angle $\alpha$ between initial and final cross-sections of the die. By varying these parameters, one can change strain intensity in different zones. In particular, profiles with a rectangular cross-section increase strain in zones 1 and 2 while decreasing it in zones 3 and 4 . If the profile contour contains circular arcs about the direction of extrusion, this leads to the opposite effect, i.e. it decreases strain in zones 1 and 2 while increasing it in zones 3 and 4 .

The presence of two new planes of controllable shear, different from those in ECAP, provides additional means for forming material microstructure and properties. For example, one can create equiaxed fragments using TE as well as using the combination of TE with ECAP. Intensification of the shear in zones 1 and 2 creates a microstructure with pronounced anisotropic properties. 
Deformation zones 3 and 4 form a vortex-like flow, which stretches metal particles. The stretching increases with subsequent TE passes as long as the dies have the same direction (all clockwise or all counter-clockwise). Passes with alternating directions create folds. Alternating stretching and folding lead to mixing as in Smale's horseshoe [19]. The capability for severe mixing is a distinguishing characteristic of TE, which can be used for homogenization of composite materials (Fig. 5), intensification of mechanochemical reactions, etc.

Zone 4 allows one to obtain very interesting mixing-related effects, due to the following factors. First, this is the zone of the most severe shear leading to formation of folds not only on the macro but also on the microlevel (Fig. 4b). As mentioned before, this leads to mixing according to the Smale's horseshoe mechanism. Also, since the surface area of the specimen first increases by $50 \%$ and then returns to its original size as the specimen goes through the die, the material in the core and on the surface mix. This can be used for mechanical impurity doping of the surface layers of the specimen.

TE opens new technological options due to the position of its shear planes. For example, TE decreases metal waste, compared with ECAP, and allows manufacturing profile specimens.

\section{Conclusion}

Twist extrusion has the following properties, which open new possibilities for forming and investigating new shapes and microstructures:

(i) As in ECAP, the deformation mode in twist extrusion is simple shear. Unlike in ECAP, there are two shear planes; one of them is perpendicular and the other is parallel to the specimen axis.

(ii) Two orientations of the twist die lead to two main routes of $\mathrm{TE}: \mathrm{CD}+\mathrm{CD}$ (or $\mathrm{CCD}+\mathrm{CCD}$ )-Route I; $\mathrm{CD}+\mathrm{CCD}$ (or $\mathrm{CCD}+\mathrm{CD})-$ Route II.

(iii) The following processes are present during twist extrusion: vortex-like flow with large strain gradient, stretching and mixing of metal particles. (iv) Twist extrusion opens new technological possibilities, for example, decreased metal waste compared with ECAP, obtaining profile specimens.

\section{References}

[1] R.Z. Valiev, Y. Estrin, Z. Horita, T.G. Langdon, M.J. Zehetbauer, Y.T. Zhu, JOM 58 (4) (2006) 33-39.

[2] N.A. Smirnova, V.I. Levit, V.I. Pilyugin, R.I. Kuznetsov, L.S. Davydova, V.A. Sazonova, Fiz. Metal. Metalloved. 61 (1986) 1170-1177.

[3] V.M. Segal, V.I. Reznikov, A.E. Drobyshevskiy, V.I. Kopylov, Russ. Metall. (Metally) 1 (1981) 99-105

[4] G.A. Salishchev, O.R. Valiakhmetov, R.M. Galeyev, J. Mater. Sci. 28 (1993) 2898-2903.

[5] Y. Saito, H. Utsunomiya, N. Tsuji, T. Sakai, Acta Mater. 47 (1999) 579-583.

[6] J.Y. Huang, Y.T. Zhu, H.G. Jiang, T.C. Lowe, Acta Mater. 49 (2001) 14971505.

[7] Ya.E. Beygelzimer, V.N. Varyukhin, S.G. Synkov, A.N. Sapronov, V.G. Synkov, Phys. Technol. High-Press. 9 (1999) 109-111.

[8] Y. Beygelzimer, D. Orlov, V. Varyukhin, in: YT, Zhu, et al. (Eds.), Proceedings of the Second International Symposium on Ultrafine Grained Materials, The Minerals, Metals, and Materials Society, Warrendale, PA, 2002, pp. 297301.

[9] Y. Beygelzimer, V. Varyukhin, D. Orlov, S. Synkov, Twist Extrusion Process for Strain Accumulation, TEAN, Donetsk, Ukraine, 2003, 87p. (in Russian).

[10] Y. Beygelzimer, D. Orlov, A. Korshunov, S. Synkov, V. Varyukhin, I. Vedernikova, A. Reshetov, A. Synkov, L. Polyakov, I. Korotchenkova, Sol. Stat. Phenom. 114 (2006) 69-78.

[11] A.P. Shpak, V.N. Varyukhin, V.I. Tkatch, V.V. Maslov, Y.Y. Beygelzimer, S.G. Synkov, V.K. Nosenko, S.G. Rassolov, Mater. Sci. Eng. A 425 (2006) 172-177.

[12] V. Varyukhin, Y. Beygelzimer, S. Synkov, D. Orlov, Mater. Sci. Forum 503-504 (2006) 335-339.

[13] V.V. Stolyarov, Ya E. Beigelzimer, D.V. Orlov, R.Z. Valiev, Phys. Metal Metall. 99 (2005) 204-211.

[14] D. Orlov, A. Reshetov, A. Synkov, V. Varyukhin, D. Lotsko, O. Sirko, N. Zakharova, A. Sharovsky, V. Voropaiev, Yu. Milman, S. Synkov, in: Y.T. Zhu, V. Varyukhin (Eds.), Nanostructured Materials by High-Pressure Severe Plastic Deformation, Springer, Dordrecht, The Netherlands, 2006, pp.77-82.

[15] M. Berta, D. Orlov, P. Prangnell, Int. J. Mater. Res. 98 (2007) 200-204.

[16] S.V. Dobatkin, A.A. Kuznetsov, G.A. Salishchev, Y.Y. Beygelzimer, D.V. Orlov, Metalurgiya 45 (2006) 245-251.

[17] P.W. Bridgman, Studies in Large Plastic Flow and Fracture, with Special Emphasis on the Effects of Hydrostatic Pressure, Harvard University Press, Cambridge, MA, USA, 1952, $444 \mathrm{p}$.

[18] Y. Beygelzimer, Mech. Mater. 37 (2005) 753-767.

[19] J.M. Ottino, The Kinematics of Mixing: Stretching, Chaos, and Transport, Cambridge University Press, Cambridge and New York, 1989, 375 p. 